\title{
Avant propos. Entre fait et fiction...
}

\section{(2) OpenEdition \\ Journals}

Édition électronique

URL : https://journals.openedition.org/ml/242

DOI : $10.4000 / \mathrm{ml} .242$

ISSN : 2274-0511

Éditeur

Association Modèles linguistiques

Édition imprimée

Date de publication : 1 janvier 2012

Pagination : 7-10

Référence électronique

«Avant propos. Entre fait et fiction... », Modèles linguistiques [En ligne], 65 | 2012, mis en ligne le 26 janvier 2013, consulté le 01 juillet 2021. URL : http://journals.openedition.org/ml/242 ; DOI : https:// doi.org/10.4000/ml.242 


\section{Avant propos}

\section{Entre fait et fiction...}

Le présent numéro de Modèles linguistiques s'articule autour d'une problématique de type narratologique. Il s'agit de questionner la pertinence du critère qui répartit les récits en deux grandes catégories suivant la nature réelle ou imaginaire des faits racontés : les récits factuels, d'un côté, et les récits fictionnels, de l'autre.

Il nous semble que ce critère surestime l'importance du statut ontologique des faits racontés au détriment de ce qui caractérise vraiment le récit et qui devrait être prioritairement pris en compte dans sa définition : son appartenance au langage, son incapacité d'accéder à l'existence en dehors d'un acte de parole, celui par lequel un narrateur articule verbalement une série de faits et permet à ceux qui l'écoutent de prendre acte de la manière dont une histoire (peu importe si elle est réelle ou imaginaire) s'est déroulée. Méconnaître l'importance de cette médiation narrative signifie passer complètement à côté de l'essence du récit et donner une lecture forcément simpliste des formes à travers lesquelles s'articule la phénoménologie des genres narratifs.

Que l'opposition entre récits factuels et récits fictionnels ne soit pas apte à circonscrire et caractériser la complexité de l'écriture narrative dans toute la variété de ses manifestations concrètes est confirmé par le fait que la nature factuelle ou fictionnelle d'une histoire n'est pas facile à déterminer, y compris lorsque le récit qui nous raconte cette histoire se présente ouvertement comme factuel ou fictionnel. Peut-on vraiment être sûr que tout ce que racontent les récits factuels est vrai ? Peut-on vraiment qualifier de faux tout ce que racontent les récits fictionnels ? Il semblerait à tout le moins hasardeux de répondre par l'affirmative au vu des incertitudes qui entourent, aujourd'hui encore, certaines œuvres de la littérature narrative, dont on ne sait pas dire exactement si elles appartiennent aux genres factuels ou aux genres fictionnels.

Dans quelle catégorie ranger les poèmes homériques ? Dans la catégorie des récits qui racontent des choses réelles comme l'auraient sûrement affirmé leurs premiers auditeurs ? Ou dans la catégorie des récits fictionnels comme l'exigeraient certainement les lecteurs d'aujourd'hui? 
L'histoire de la réception des textes narratifs est traversée, de part en part, par des erreurs d'appréciation qui ont amené les lecteurs à reclasser dans la catégorie des récits fictionnels des récits qui avaient été initialement reçus comme factuels ${ }^{1}$ ou, à l'inverse, à reclasser comme factuels des récits qui avaient été reçus (ou présentés), à leur apparition, comme fictionnels ${ }^{2}$. Comment expliquer ces reclassements si la nature factuelle et fictionnelle des faits racontés par les genres narratifs était si facile à déterminer ?

Qu'il soit difficile de se prononcer sur la nature réelle ou imaginaire des faits racontés dans un récit vient aussi du fait que beaucoup d'auteurs se plaisent à entretenir le doute sur la nature de leur récit et articulent volontairement des styles d'écriture codés comme factuels ou fictionnels à l'intérieur d'un même texte. Combien de romans épistolaires, biographiques, historiques ont-ils l'apparence d'un vrai recueil de lettres, d'un vrai journal intime ou d'une vraie biographie ? Et combien de récits journalistiques, historiques, autobiographiques nous donnent-ils l'impression de lire, non pas une histoire vraie, mais une histoire imaginaire, enjolivée de toutes les figures de style, de narration et de pensée qui sont propres aux genres fictionnels ? Dès lors, on voit mal l'intérêt qu'il pourrait y avoir à axer la compréhension du récit autour d'une distinction si floue et si difficile à établir.

Mais il y a un dernier aspect qui nous amène à douter de l'utilité heuristique de l'opposition entre factuel et fictionnel en tant que critère de classement des récits. Il est fort douteux, en effet, que l'on puisse expliquer la genèse du plaisir que l'on prend à écouter ou lire une histoire à partir de la nature factuelle ou fictionnelle des faits racontés. Certes, la psychanalyse nous apprend que les états psychiques placés sous le contrôle du principe de réalité sont moins propices au plaisir que ne le sont les états psychiques dépendant d'activités imaginaires comme le rêve, les fantaisies diurnes (Tagtraum), les hallucinations, dont le caractère irréel est évident. Il semblerait donc légitime d'en conclure qu'il est plus facile d'accéder à une forme de jouissance esthétique par l'intermédiaire des récits fictionnels que par l'intermédiaire des récits factuels. Mais ce constat ne doit pas nous faire écarter comme impossible le fait de pouvoir prendre

1. C'est le cas de Marbot (1981), roman de Wolfang Hildesheimer (1916-1991), considéré, pendant un certain temps, par les lecteurs comme une vraie biographie, alors qu'il s'agit d'une biographie fictive, c'est-à-dire d'un roman. Voir, à ce sujet : J.-M. Schaeffer, Pourquoi la fiction ? , Paris, Seuil, 1999, pp. 133-145.

2. Voir dans ce volume l'article de Marine Bernot sur Ford Madox Ford (1873-1939). 
plaisir à la lecture d'un récit relatant des faits réels. Si tel n'était pas le cas si l'intérêt qui nous pousse à lire un récit journalistique, historique, biographique était uniquement la recherche d'une information - les genres narratifs contemporains (y compris ceux qui occupent une place de plus en plus importante dans la grille des programmes des chaînes de télévision actuelles ${ }^{3}$ ) ne mettraient pas autant de soin à essayer de se donner les apparences d'un récit fictionnel (le docu-fiction) ${ }^{4}$. Faits réels ou imaginaires ont donc tous les deux les mêmes chances d'assouvir cette irrésistible soif $d^{\prime}$ histoires qui caractérise l'espèce fabulatrice ${ }^{5}$ que nous sommes. Que certains récits y parviennent mieux que d'autres ne dépend pas tant du statut ontologique de l'histoire racontée (histoire réelle vs histoire inventée) que de l'art déployé par le narrateur dans l'articulation des deux temporalités constitutives de tout récit (temps raconté et temps du raconté) ainsi que de l'interaction qui s'établit entre les structures du texte et les structures attendues par l'interprète dans ce jeu d'anticipations et de développements non-attendus qui caractérise le dialogue entre l'auteur d'un récit et son public. C'est à la mise à jour des règles de cet art et de cette interaction - des règles qui surdéterminent et les récits factuels et les récits fictionnels - que doit se consacrer une narratologie soucieuse de dépasser la dichotomie réel/imaginaire à l'intérieur de laquelle la compréhension du récit a eu tendance, ces derniers temps, à s'enfermer.

Pour résumer, les raisons qui justifient ce nouveau questionnement du problème du récit en dehors de la dichotomie factuel/fictionnel sont à la fois méthodologiques, narratologiques et esthétiques : méthodologiques, parce que l'opposition entre récits factuels et récits fictionnels est difficile à établir ; narratologiques, parce que le récit est un fait de langage et le langage se joue de l'opposition entre ce qui est réel et ce qui est imaginaire (le propre du langage est de signifier, non pas de nous dire si une chose existe ou n'existe pas), esthétiques, parce que l'opposition factuel/fictionnel est inapte à expliquer le plaisir que nous prenons à lire ou écouter une histoire.

Le numéro s'organise autour des axes thématiques suivants :

(i) Réflexion théorique sur la fiction dans le cadre d'une théorie narratologique attentive à la dimension esthétique de la narration et aux mécanismes rhétoriques et herméneutiques qui rendent possible

3. F. Jost, La télévision du quotidien. Entre réalité et fiction, Paris, De Boeck Université/INA, « Médias Recherches », 2001.

4. Isabelle Veyrat-Masson, Télévision et histoire, la confusion des genres. Docudramas, docufictions et fictions du réel, Paris, De Boeck Université/INA, 2008.

5. Nancy Huston, L'espèce fabulatrice, Arles, Actes Sud, 2008. 
l'émergence du plaisir qui accompagne l'acte de raconter ou de lire (écouter) un récit.

(ii) Mise à l'épreuve de l'opposition factuel/fictionnel à travers l'étude de toute une série de récits où les frontières entre réel et imaginaire sont plutôt floues et imprécises, l'histoire racontée basculant souvent du réel à l'imaginaire et de l'imaginaire au réel.

Alessandro Leiduan essaie de décrire l'expérience de suspension temporelle que déclenche la lecture d'un récit à partir des outils analytiques de la linguistique guillaumienne.

Raphaël Baroni, dans l'entretien qui lui est consacré, fait le point sur les développements récents de la narratologie et expose son point de vue sur le phénomène du panfictionnalisme.

Dairine Ó Kelly propose une analyse critique de la technologie du discours narratif, c'est-à-dire restrictivement du récit, selon Genette. Après avoir constaté que son étude du temps dans les récits de fiction (ordre, durée, fréquence des événements) ne repose sur aucun fondement linguistique, elle passe au crible diverses distinctions qui ont fait fortune, comme par exemple celle du «singulatif » et de l'« itératif ». L'examen d'un long extrait de Proust montre que Genette n'a en fait rien compris aux « intermittences du passé ».

Marine Bernot aborde, par le biais de la notion d' "impressionnisme », le style d'écriture mi-factuel, mi-fictionnel de Ford Madox Ford, écrivain qui qualifiait ses œuvres à caractère autobiographique de « romans ».

Philippe Duley s'interroge sur les liens entre journalisme et fiction en mettant en lumière les points de convergence entre l'écriture journalistique et l'écriture fictionnelle, mais en soulignant aussi les aspects qui font de ces deux activités deux pratiques narratives pour ainsi dire uniques dans leur genre.

Pierre-François Peirano étudie l'imbrication étroite entre la fiction et le réel dans Le Journal de Julius Rodman ainsi que dans l'imagination collective de la société américaine de la fin du XIXe siècle.

Les auteurs remercient vivement Nicole Mausset et Monique Léonard du comité de lecture pour leur relecture scrupuleuse.

Modèles linguistiques 